\title{
Mild cases of Covid-19 in children. is it really true or a mirage?
}

\author{
Mandal A. ${ }^{1 *}$, Kondekar S. ${ }^{2}$, Bothara R. ${ }^{3}$ \\ DOI: https://doi.org/10.17511/ijpr.2020.i08.10
}

1* Abhishek Mandal, Final MBBS year, TNMC and Nair Hospital, Mumbai, Maharashtra, India.

2 Santosh Kondekar, Associate professor, TNMC and Nair Hospital, Mumbai, Maharashtra, India.

${ }^{3}$ Rishi Bothara, Final MBBS year, TNMC and Nair Hospital, Mumbai, Maharashtra, India.

To date, very few data on pediatric COVID 19 cases have been published, and most reports originate from China. There is an urgent need to understand why the course of the coronavirus that started in late 2019 is affecting different groups of individuals with varying severity during the ongoing global pandemic. Globally, the greatest burden of most infectious diseases, especially infections of the respiratory system are borne by children, hence the low burden of COVID 19 in children has been viewed by many as surprising. This article will provide insights regarding the role of constitutional higher trained immunity in children, physiological advantages of children, higher insulin secretion, higher ACE2 levels, better antioxidant potential, and absence of high-risk factors in protecting children against severe COVID 19 infections.

Keywords: SARS-CoV2, Children, ACE2, Trained immunity, Antioxidant

Corresponding Author

Abhishek Mandal, Final MBBS year, TNMC and Nair Hospital, Mumbai, Maharashtra, India. Email: abhishek.mandal98@gmail.com
How to Cite this Article

Mandal A, Kondekar S, Bothara R. Mild cases of Covid-19 in children. is it really true or a mirage?. Pediatric Rev Int J Pediatr Res. 2020;7(8):446-451. Available From

https://pediatrics.medresearch.in/index.php/ijpr/arti cle/view/644
To Browse

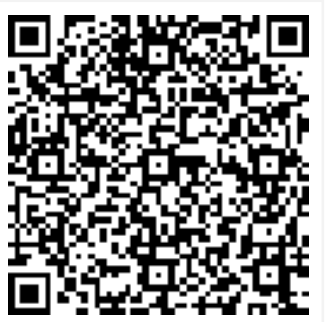

Manuscript Received 2020-12-11

Conflict of Interest No
Review Round 1 2020-12-21

Funding $\mathrm{Nil}$
Review Round 2 2020-12-25

Ethical Approval Yes
Review Round 3

Plagiarism X-checker $7 \%$
Accepted 2020-12-28

Note

(C) 2020 by Abhishek Mandal, Santosh Kondekar, Rishi Bothara and Published by Siddharth Health Research and Social Welfare Society. This is an Open Access article licensed under a Creative Commons Attribution 4.0 International License https://creativecommons.org/licenses/by/4.0/ unported [CC BY 4.0]. 


\section{Introduction}

Wuhan, China notified to WHO in late December 2019 of an unusual cluster of pneumonia cases. The disease later termed COVID 19, spread quickly beyond the borders of China, with the first cases in Europe being recorded on Jan 25, 2020. SARS-CoV2 is the causative agent for COVID 19 infections in humans. This is a positive sense, single-stranded RNA enveloped virus, comprising of glycoprotein spikes on the outer surface, which mediates its entry into the host cell.

ACE2 receptor serves as a molecular target for the novel coronavirus, to enter the target cell, by interacting with the viral glycoprotein spikes. Also, ACE2 has lung-protective effects by limiting angiotensin 2 mediated pulmonary capillary leaks and inflammation. It leads to a cascade of immune responses in the host causing acute respiratory distress syndrome. In this article, it was aimed to discuss the various hypotheses and possible reasons as to why children have milder symptoms as compared to adults.

\section{There are many potential factors protecting children against severe SARS CoV2 infection:}

1. Prevention of virus exposure: Probably strict quarantine measures and closing of potential hotspots for pediatric infections like schools and daycare centers. As the movement of children is highly restricted in these times, most of the infections are only spread from infected family members [1].

\section{Appropriate infection handling: Children} supposedly have trained immunity which means that it is the functional reprogramming of the innate immune cells to a more activated state following initial antigen stimulation (infections or vaccination) through metabolic reprogramming and epigenetic changes (acetylation and demethylation leading to enhanced transcription of Interleukins and tumor necrosis factor) [1-4]. It is known that children uniquely own an active thymus, a lymphatic organ in which the formed T-cells mature, develop and reproduce to fight intracellular pathogens throughout childhood. Thus, there is a greater number of naïve $T$ cells that can be programmed to protect them against the disease [5]. Another hypothesis suggests that these naïve $T$ cells program into memory $T$ cells which play an important role in conferring protection to children against COVID-19 [6].
Thus, based on trained immunity it has also been postulated that countries with routine BCG vaccination have less COVID 19 related morbidity and mortality [1]. In a study, it has been found that 30 amino acid residues share similarities between the Spike (S) glycoprotein of the SARS-CoV2 and the fusion (F1) glycoprotein of the Measles virus as well as with the envelope (E1) glycoprotein of the Rubella virus [7].

It is now known that the Spike (S) glycoprotein of the SARS-CoV2 is a key immunogenic protein of SARS-CoV2 which induces host immune response [8] and this short 30 amino acid residues of the fusion (F1) glycoprotein of Measles virus as well as with the envelope (E1) glycoprotein of the Rubella virus appear to have an epitope property and is involved in antibody production [9].

The fusion (F1) glycoprotein of the measles virus induces the production of neutralizing antibodies [10] and the envelope (E1) glycoprotein of the Rubella also has a receptor-binding function, so it is the main antigen and sole target of neutralizing antibodies against the rubella vaccine [11]. Thus, trained immunity due to routine live vaccines like $M M R$, influenza, and frequent viral infections could be an important protective mechanism $[1,7]$.

Also, other coronavirus infections are common causes of upper respiratory tract infections in children, the protective role of antibodies from other coronavirus infections against SARS CoV2 cannot be ruled out. It has been reported that common circulating human coronaviruses can be isolated from $4 \%-8 \%$ of children hospitalized for acute respiratory tract infections and from $8 \%$ of children in an ambulatory setting [2].

It can also be postulated that co-infection with human coronavirus NL-63, can prevent the occurrence of COVID-19 infection, as NL-63 binds to the same receptor as SARS CoV2 and is less pathogenic [12].

3. Developmental changes in Immunity: The elderly are predisposed to increased incidence and severity of Acute lung injury because of agingrelated immune senescence. Multiple innate immunity aberrations have been reported in the elderly which includes decreased TLR induction, reduced dendritic cell number and function, decreased macrophage and neutrophil function, upregulation of proinflammatory cytokines, reduced NK-cell activity, decreased $\mathrm{T}$ cell proliferation and number [1]. 
4. Effects of Lung Development: Lungs gradually have impaired regenerative potential while aging. Since children have greater upper airway resistance, aerosol particles deposit more in the tracheobronchial tree than alveoli, which could lead to less pneumonia and more bronchiolitis-like infections with SARS-CoV2 infection in children [1].

Also, an adult has 500 million alveoli compared to only 3 million alveoli in a newborn which may translate into less severe immune reaction compared to adults as fewer alveoli mean fewer type 2 cells. Studies show that the cellular serine protease TMRRSS2 is also required to allow the entry of coronavirus into host cells and type 2 cells express TMRRSS2 [13].

Androgenic hormones upregulate this gene TMRRSS2, which may show the low incidence of COVID 19 infections in the prepubertal age group [14]. In adults, ventilation of alveoli also occurs via alternate pathways like alveolar pores of Kohn, interbronchiolar Martin's channels, and bronchioalveolar Lambert's channels and this is known as collateral ventilation.

In children, these pathways are said to be developed in 5 to 6 years and it can also be speculated that the virus may also spread through these collateral pathways and its absence may prevent the excessive spread of the virus in children [14]. Children also have larger alveolar ventilation and functional residual capacity is smaller which may translate into a higher alveolar clearance in children as compared to adults.

5. ACE 2 regulation: There is a supposedly high ACE 2 expression in the pediatric population which metabolizes angiotensin 2 and inhibits its proinflammatory, fibrotic effects. Since there is a downregulation of ACE2 expression, there might be a positive relationship between the concentration of angiotensin 2 in the body and the severity of SARS CoV2 mediated lung injury $[1,4]$. As per the enzymatic analysis, the levels of ACE2 in children are $13-100 \mathrm{U} / \mathrm{L}$, unlike the levels of adults which is 9-69 U/L [4]. The circulating soluble ACE2 levels aid children and asymptomatic individuals, to counteract the spreading of the virus in the body, by buffering effect, more like neutralizing antibodies.

6. Evolutionary link to disease severity: [3] Bats are known to be the natural reservoir of coronaviruses and they chronically shed these viruses without any major disease in the host animal.
From this perspective, both children aand bats are similar in that sense as they carry and shed the virus without any ill effects. Both bats and children have one thing in common i.e. high metabolic rate, which is required for growth and development in children, and the cost of the flight in bats. It has been found that a high metabolic rate is associated with high levels of oxidative stress and antioxidant capacity in both children and bats.

Both bats and children have a high level of melatonin secretion secondary to a significant amount of time spent in darkness or sleep, respectively. Melatonin has significant immunomodulatory effects and is also a very potent antioxidant. It can be said that Coronaviruses probably co-evolved with bats over long periods.

Since it is known that bat tissues have a high antioxidant capacity, it is logical to speculate that co-evolution of coronaviruses with bats led to the adaptation to an environment that is high in oxidative stress and total antioxidant capacity with a balanced redox state.

Under these conditions, SARS CoV2 is likely to create mild infections whether it is in children or bats. If low metabolism is coupled with low antioxidant capacity, then SARS CoV2 infections are likely to become severe in these kinds of biological environments. ACE2 also reduced oxidative stress and children have supposedly higher expression of ACE2. The image-related difference in endogenous antioxidant enzymes like super oxidase dismutase 3 may be a factor deciding severity in populations.

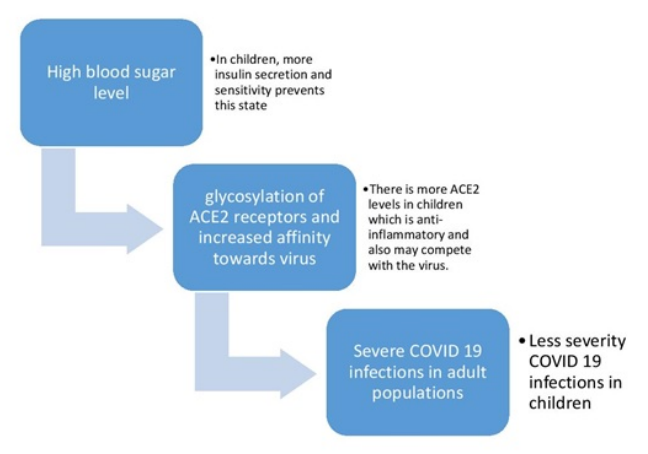

Fig-1: Role of blood sugar levels and ACE2 levels in the severity of Covid-19 infections in children 


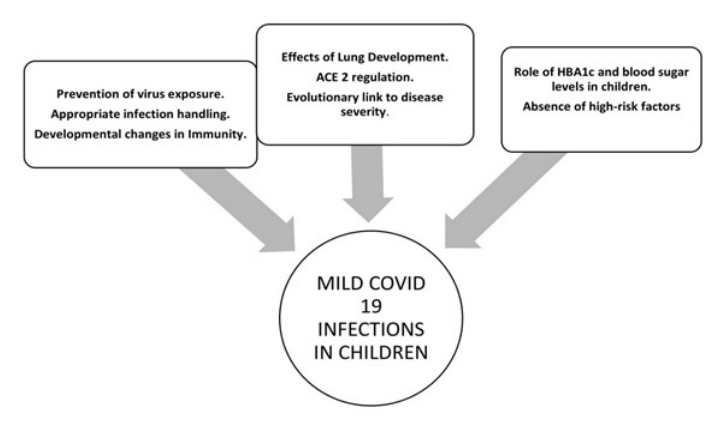

Fig-2: Mild Covid-19 infections in children.

Thus, it has been clear that from evidence, the antioxidant capacity and the antioxidant response potential of the host are two of the key determinants of SARS CoV2 infection outcome. The higher antioxidant capacity of children and young adults combined with their higher ACE2 expression is one of the likely explanations of the low incidence of severe infections in these age groups.

7. Role of HBA1c and blood sugar levels in children: It is known that with advancing age, the tissue sensitivity to insulin decreases which further results in insulin resistance $[15,16,17]$. There is the robust secretion of insulin from the pancreas and also good sensitivity of tissue to insulin which probably explains the low levels of HBA1c levels in children [18].

Now the two events occurring i.e. the mortality related to COVID 19 increases with age and the average $\mathrm{HbA1C}$ of the general population also increases with age, which has been postulated to be causally related to each other [19]. It has been proposed that hyperglycemia in both diabetic and non-diabetic patients may lead to the glycosylation of ACE2 receptors, which may increase the affinity towards SARS CoV2 resulting in severe infection [20].

Since children have better insulin sensitivity, thus lesser HBA1c levels which indicates fewer chances of the hyperglycemic state which may also contribute to protecting children from severe infections of COVID 19.

8. Absence of high-risk factors: There is the absence of any aging-related comorbidities and less degree of smoking, obesity, hypertension.

\section{Future Perspectives}

Although mild symptomatic can be seen in cases in a majority of the pediatric population, it can't be certain that this is the final result of COVID 19 in the pediatric population because of the lack of large-scale data of this infection occurring in children. Mild symptoms in children and high chances of being asymptomatic carriers may further alleviate the risk of spreading the virus in a highly vulnerable population. Thus, keeping this in mind, it is suggested that potential pediatric hotspots like schools and parks should be opened only after taking the strictest preventive measures and proper education of children to understand the importance of these measures. Also, these insights into the pathophysiology behind mild infections in children can further guide and help researchers to look for specific treatment modalities for this deadly virus.

\section{Conclusion}

Hence, it can be speculated that high ACE2 expression, receptors concentrations, trained immunity, constitutionally higher lymphocyte count in children, vaccinations against various respiratory viruses, physiological advantages, better antioxidant potential and concentration, lower HBA1c levels, and better insulin sensitivity may be the probable reasons behind the mild COVID 19 presentation in children.

\section{Reference}

01. Dhochak N, Singhal T, Kabra SK, Lodha R. Pathophysiology of COVID-19- Why Children Fare Better than Adults?. Indian J Pediatr. 2020;1.

doi: $\quad 10.1007 / \mathrm{s} 12098-020-03322-y \quad$ [Crossref]

02. Zimmermann $P$, Curtis N. Coronavirus infections in children including COVID-19- an overview of the epidemiology, clinical features, diagnosis, treatment, and prevention options in children. Pediatr Infect Dis J. 2020;39(5)355-368.

doi: $10.1097 /$ INF.0000000000002660 [Crossref]

03. Keles ES. Mild SARS-COV-2 infections in children might be based on evolutionary biology and linked with host reactive oxidative stress (ROS) and antioxidant capabilities. New Microbes New Infect. $2020 ; 36 ; 100723$.

doi: $\quad 10.1016 /$ j.nmni.2020.100723 [Crossref] 
04. Behl T, Kaur I, Bungau S, Kumar A, Uddin MS, Kumar $C$, et al. The dual impact of ACE2 in COVID-19 and ironical actions in geriatrics and pediatrics with possible therapeutic solutions. Life Sci. $2020 ; 118075$.

doi: $10.1016 / j . I f s .2020 .118075$ [Crossref]

05. Ahmadpoor P, Rostaing L. Why the immune system fails to mount an adaptive immune response to a Covid-19 infection. Transplant International. 2020;33(7)824-825. doi: $10.1111 /$ tri.13611 [Crossref]

06. Sabir DK, Sidiq KR, Ali SM. Current speculations on the low incidence rate of the COVID-19 among children. Int $\mathrm{J}$ School Health. 2020;7(2)61-62.

[Crossref]

07. Sidiq KR, Sabir DK, Ali SM, Kodzius R. Does Early Childhood Vaccination Protect Against COVID-19?. Front Mol Biosci. 2020;7;120. doi: $10.3389 /$ fmolb.2020.00120 [Crossref]

08. Ahmed SF, Quadeer AA, McKay MR. Preliminary identification of potential vaccine targets for the COVID-19 coronavirus (SARS-CoV-2) based on SARS-CoV immunological studies. Viruses. 2020;12(3)254.

doi: $10.3390 / v 12030254$ [Crossref]

09. Larsen JE, Lund O, Nielsen M. Improved method for predicting linear B-cell epitopes. Immunome Res. $2006 ; 2(1) 1-7$.

doi: $10.1186 / 1745-7580-2-2$ [Crossref]

10. Malvoisin ET, Wild FA. Contribution of measles virus fusion protein in protective immunity- anti$F$ monoclonal antibodies neutralize virus infectivity and protect mice against challenge. J Virol. 1990;64(10)5160-5162.

doi: $10.1128 / J V I .64 .10 .5160-5162.1990$ [Crossref]

11. DuBois RM, Vaney MC, Tortorici MA, Al Kurdi R, Barba-Spaeth G, Krey $T$, et al. Functional and evolutionary insight from the crystal structure of rubella virus protein E1. Nature. 2013;493(7433)552-556.

doi: $10.1038 /$ nature11741 [Crossref]
12. Liu DX, Liang JQ, Fung TS. Human Coronavirus229E, -OC43, -NL63, and -HKU1. Reference Module Life Sci. 2020.

doi: $\quad 10.1016 /$ B978-0-12-809633-8.21501-X [Crossref]

13. Hoffmann $M$, Kleine-Weber $H$, Schroeder $S$, Krüger N, Herrler T, Erichsen $S$, et al. SARSCOV-2 cell entry depends on ACE2 and TMPRSS2 and is blocked by a clinically proven protease inhibitor. Cell. 2020;181(2)271-280.

e8. doi: 10.1016/j.cell.2020.02.052 [Crossref]

14. Yavuz S, Kesici S, Bayrakci B. Physiological advantages of children against COVID-19. Acta Paediatrica. 2020;109(8)1691.

doi: 10.1111/apa.15410 [Crossref]

15. Pani LN, Korenda L, Meigs JB, Driver C, Chamany S, Fox CS, et al. Effect of aging on A1C levels in individuals without diabetesevidence from the Framingham Offspring Study and the National Health and Nutrition Examination Survey 2001-2004. Diabetes Care. 2008;31(10)1991-1996.

doi: $10.2337 / \mathrm{dc0} 08-0577$ [Crossref]

16. Sacks DB, Bruns DE, Goldstein DE, Maclaren NK, McDonald JM, Parrott M. Guidelines and recommendations for laboratory analysis in the diagnosis and management of diabetes mellitus. Clin Chem. 2002;48(3)436-472.

[Crossref]

17. Dubowitz N, Xue W, Long Q, Ownby JG, Olson $D E$, Barb D, et al. Aging is associated with increased HbA1c levels, independently of glucose levels and insulin resistance, and also with decreased HbA1c diagnostic specificity. Diab Med. 2014;31(8)927-935.

doi: $10.1111 /$ dme.12459 [Crossref]

18. Roth J, Müller N, Lehmann T, Heinemann L, Wolf G, Müller UA. HbA1c and age in non-diabetic subjects- an ignored association?. Experiment Clin Endocrinol Diab. 2016;124(10)637-642.

doi: $10.1055 / \mathrm{s}-0042-105440$ [Crossref]

19. Kalbhande JG, Kuldeep V. Use of Insulin in treatment of COVID 19- a proposal to explore feasibility. J Med Sci Clin Res. 2020;8(7).

doi: $10.18535 / j m s c r / v 8 i 7.103$ [Crossref] 
20. Brufsky A. Hyperglycemia, hydroxychloroquine, and the COVID-19 pandemic. J Med Virol. 2020;92(7)770-775.

doi: $10.1002 / j m v .25887$ [Crossref] 